<smiles>c1ccc([Se]c2ccccn2)nc1</smiles>

However, we cannot exclude that a very small amount of IFN $\alpha / \beta$ was present in the preparations.

In the 3-4-day-old cultures of PBL $\left(10^{6}\right.$ cells $\left.\mathrm{ml}^{-1}\right)$ in RPMI 1640 medium supplemented with $10 \%$ fetal calf serum and compounds 1 or $2\left(10-90 \mu \mathrm{g} \mathrm{ml}^{-1}\right)$, stimulation of the incorporation of ${ }^{3} \mathrm{H}$-thymidine was observed. This was evident in the presence or absence of PHA $\left(1-5 \mu \mathrm{g} \mathrm{ml}^{-1}\right)$. The observations suggest that the selenoorganic compounds may also induce synthesis of mitogenic interleukins or other cytokines (data not shown). It can be concluded that organic diselenides and Ebselen are potent IFN- $\gamma$ and TNF inducers in human PBLs but they do not stimulate IFN release in human fibroblasts. Ebselen may act as an IFN inducer after conversion to diselenide. Although the mode of action of the compounds is not known it appears to involve both adherent and non-adherent leukocytes. Because the selenoorganic compounds are undergoing clinical trials as anti-inflammatory drugs their novel IFN- $\gamma$ and TNF-inducing activity and potential immunostimulating action deserve special attention.

It is worth pointing out that IFN- $\gamma$ has been successfully used for treating patients with rheumatoid arthritis ${ }^{17}$.

Acknowledgments. The authors thank Mrs M. Albin for titration of interferons, Dr A. Sypula and Mrs Z. Tomaszewska for preparation of leukocytes and Dr J. Czyrski for carrying out the mitogenic assays. The study was supported by grants of the Polish Academy of Sciences (CPBP 06.01 and CPBR-3/3)

1 Mannering, G. J., and Deloria, L. B., A. Rev. Pharmac. Toxic. 26 (1986) 455 .

2 Johnson, H. A., in: Mechanisms of Interferon Actions, vol. II, p. 60. Ed. L. M. Pfeffer. CRC Press Inc., Boca Raton, Florida 1987.

3 Antonelli, G., Dianzani, F., Van Damme, J., Amicucci, P., De Marco, F., and Cefaro, A., Cell. Immun. 113 (1988) 376.

4 Salas, M., and Kirchner, H., Clin. Immun. Immunopath. 45 (1987) 139.

5 Syper, L., and Młochowski, J, Tetrahedron Lett. 43 (1987) 207.

6 Syper, L., and Młochowski, J., Tetrahedron Lett. 44 (1988) 6119.

7 Parnham, M. J., and Graf, E., Biochem. Pharmac. 36 (1987) 3095.

8 Müller, A., Gabriel, H., Sies, H., Trelinden, R., Fischer, H., and Römer, A., Biochem. Pharmac 37 (1988) 1103.

9 Lambert, C., Cantineau, R., Christiaens, L., Biedermann, J., and Dereu, N., Bull. Soc. chim. Belg. 96 (1987) 383.

10 Van Dyke, T. E., Braswell, L., and Offenbacher, S., Agents Actions 19 (1986) 376.

11 Inglot, A. D., Piasecki, E., Szulc, B., Groehlich, B., Dobracki, W., GTadysz, A., Leszeh, J., Szklarz, E., and Seiborski, R. Archs Immun. Ther. exp. 36 (1988) 439.

12 Standardization of Interferons. Annex 1. Report of WHO informal consultation, pp. 37-87. WHO Technical Report Series No 771 (1988).

13 Cantell, K., Hirvonen, S., and Kauppinen, H. L., Meth. Enzymol. 119 (1986) 54.

14 Hart, P. H., Whitty, G. A., Piccoli, D. S., and Hamilton, J.A., J. Immun. 141 (1988) 1516.

15 Inglot, A. D., J. gen. Virol. 4 (1969) 203.

16 Inglot, A. D., Archs Immun. Ther. exp. 31 (1983) 601

17 Obert, H. J., Annual Meeting of the International Society for Interferon Research. Abstracts. Kyoto, 14-18 Nov. 1988 p. 122 and Florence, $22-27$ Oct. 1989 , p. $\$ 130$.

$0014-4754 / 90 / 030308-04 \$ 1.50+0.20 / 0$

(C) Birkhäuser Verlag Basel, 1990

\title{
Microtubule inhibitors block the morphological changes induced in Drosophila blood cells by a parasitoid wasp factor
}

\author{
R. M. Rizki and T. M. Rizki
}

Department of Biology, The University of Michigan, Ann Arbor (Michigan 48109, USA) Received 14 June 1989; accepted 18 August 1989

Summary. The shape change of Drosophila melanogaster blood cells (lamellocytes) from discoidal to bipolar that is caused by a factor from the female parasitoid Leptopilina heterotoma is blocked by the tubulin inhibitors vinblastine and vincristine in vitro. The actin inhibitor, cytochalasin B, causes arborization of Drosophila lamellocytes and acts synergistically with the wasp factor to alter lamellocyte morphology. Lamellocyte arborization induced by cytochalasin B is blocked by simultaneous treatment with vinblastine. These observations indicate that the changes in lamellocyte shape induced by both the wasp factor and cytochalasin B require microtubule assembly.

Key words. Drosophila melanogaster; Leptopilina heterotoma; parasitoid; blood cells; vinblastine; vincristine; cytochalasin B; cytoskeleton.

The ability of Drosophila melanogaster blood cells to encapsulate foreign objects is destroyed by the parasitoid Leptopilina heterotoma ${ }^{1}$. The female wasp injects a factor, named lamellolysin, along with its eggs that selectively incapacitates the capsule-forming blood cells of Drosophila. The destruction of these hemocytes, lamel- locytes $^{2}$, is an orderly process in which the thin, discoidal cells become bipolar and shed their elongating cytoplasmic ends. The morphologically-altered lamellocytes are no longer adhesive so they are unable to layer around the parasitoid eggs to contain them and the eggs develop undisturbed in the host hemocoel. 
The disposition of the microtubules in discoidal lamellocytes and in lamellocytes from parasitized larvae was examined by indirect immunofluorescence using antitubulin ${ }^{1}$. The microtubules in normal lamellocytes radiate from the vicinity of the nucleus toward the cell periphery, resembling the dispersion of microtubules found in tissue culture cells ${ }^{3,4}$. In lamellocytes affected by the wasp factor, the microtubules assume a new orientation parallel to the elongating axis of the cells. If the lamellocyte elongation induced by lamellolysin depends on the depolymerization and repolymerization of tubulin, then drugs that disrupt tubulin assembly should block the change in cell shape. On the other hand, lamellocyte elongation stimulated by lamellolysin may force the microtubules along the elongating axis and the elongation process may remain unaffected by tubulin inhibitors. The present study was undertaken to determine what effects, if any, inhibitors of cytoskeletal components have on the cell elongation induced by lamellolysin.

Lamellolysin is found in the reservoir of an accessory gland of the female reproductive system. Injection of reservoir fluid into Drosophila larvae causes lamellocyte elongation in vivo ${ }^{1}$, and we recently developed methods for studying the effects of lamellolysin on Drosophila hemocytes in vitro ${ }^{5}$. Drosophila hemocytes in vitro were treated with inhibitors for tubulin and actin polymerization ${ }^{6,7}$ together with reservoir fluid from $L$. heterotoma females, and the lamellocytes in these samples were compared with lamellocytes in control samples containing either reservoir fluid or inhibitor alone.

\section{Materials and methods}

The source of hemocytes was late third instar (8-10-dayold) giant heterozygote larvae ${ }^{5}$ (ysc gt $t^{\mathrm{xII}} v^{8 \mathrm{lix}} t u-S z^{\mathrm{ts}} / g t$ $\left.w^{\text {a }} t u-S z^{\text {ts }}\right)$ that carried the $t u-S z^{\text {ts }}$ melanotic tumor gene. For explanation of gene symbols, see Lindsley and Grell $^{9}$. Larvae of this strain have a large volume of hemolymph with an abundance of lamellocytes. The larvae were raised on live Fleischmann's yeast growing on cream of wheat $/$ molasses medium at $27^{\circ} \mathrm{C}$. Hemolymph was collected in phosphate-buffered saline for Drosophila cells ${ }^{10}$ containing $0.07 \%$ phenylthiourea to prevent the darkening that occurs if activation of phenoloxidases is not blocked. Aliquots of a single, pooled hemolymph sample were dispensed to sterile Sykes chambers as described previously ${ }^{5}$ so that each chamber contained hemocytes from about 1.5 larvae in a total incubation volume of $140 \mu 1$. Experiments were conducted such that control and treated hemocytes were obtained from the same hemocyte sample.

The Leiden strain of $L$. heterotoma was used for collecting reservoir fluid. Adult females (4-6 weeks old) were rinsed in $95 \%$ alcohol and sterile water before dissection. Reservoir fluid was collected in saline, and aliquots were added to the Sykes chambers so that a hemocyte sample received the reservoir fluid equivalent to that obtained from five females. Inhibitors were added to the hemocyte samples $15 \mathrm{~min}$ after the addition of the reservoir fluid. Vinblastine (VBL), vincristine sulfate (VCR) and cytochalasin B (CYB) obtained from Sigma Chemical Co. were dissolved in dimethyl sulfoxide (DMSO) and added to hemocyte samples to yield a final concentration of $2 \mu \mathrm{g} / \mathrm{ml}$ of VBL and VCR and $10 \mu \mathrm{g} / \mathrm{ml}$ of CYB. The Sykes chambers were kept at room temperature (22$23^{\circ} \mathrm{C}$ ). Hemocytes in the chambers were examined periodically beginning at $30 \mathrm{~min}$ and ending at $5 \mathrm{~h}$ with an Olympus CK 2 inverted microscope and photographed with a $35-\mathrm{mm}$ camera.

\section{Results}

The concentration of DMSO in the hemocyte samples incubated with inhibitors ranged from $0.02 \%$ to $0.1 \%$. Therefore, control samples that contained these concentrations of DMSO, with and without reservoir fluid, were examined as well as control samples of hemocytes in buffer and hemocytes in buffer-containing reservoir fluid. Lamellocytes retained their normal discoidal morphology in the presence of DMSO, and DMSO did not interfere with the changes in lamellocyte morphology induced by lamellolysin in wasp reservoir fluid. The morphology of discoidal lamellocytes is shown in figure $1 \mathrm{~A}$. The cells have a few surface folds; otherwise their surfaces and margins are smooth. The typical elongation of lamellocytes caused by lamellolysin is shown by the lamellocyte to the left in figure $1 \mathrm{~B}$. The elongating ends of affected lamellocytes often show small, light-contrasting blebs either at or near their distal tips while the region around the nucleus generally remains wide until many hours later ${ }^{5}$. An occasional lamellocyte from a parasitized larva ${ }^{1}$ and some lamellocytes in vitro, especially when exposed to higher concentrations of lamellolysin ${ }^{5}$, develop numerous filaments from their bipolar ends and much finer filamentous extensions from other parts of the cell surface. Plasmatocytes spread against the glass surface both in the presence and absence of lamellolysin. Their morphology remained unaffected by lamellolysin.

When VBL or VCR was added to a hemocyte sample containing wasp reservoir fluid, the lamellocyte elongation caused by lamellolysin was blocked. The lamellocytes treated with these tubulin inhibitors were crinkled and this crinkling extended to the margins of the discoidal cells. Control samples of lamellocytes treated with VBL or VCR showed the same crinkled topography. Since there were no differences between lamellocytes treated with VBL or VCR, only photographs of VCRtreated cells are included in figure $1(\mathrm{C}$ and $\mathrm{D})$. Plasmatocytes spread in the presence of VBL or VCR but the surfaces of these flattened plasmatocytes did not crinkle as did the surfaces of the lamellocytes.

Lamellocytes treated with CYB generally assumed a stellate form (fig. 1E) or became arborized (fig. 2C). In 

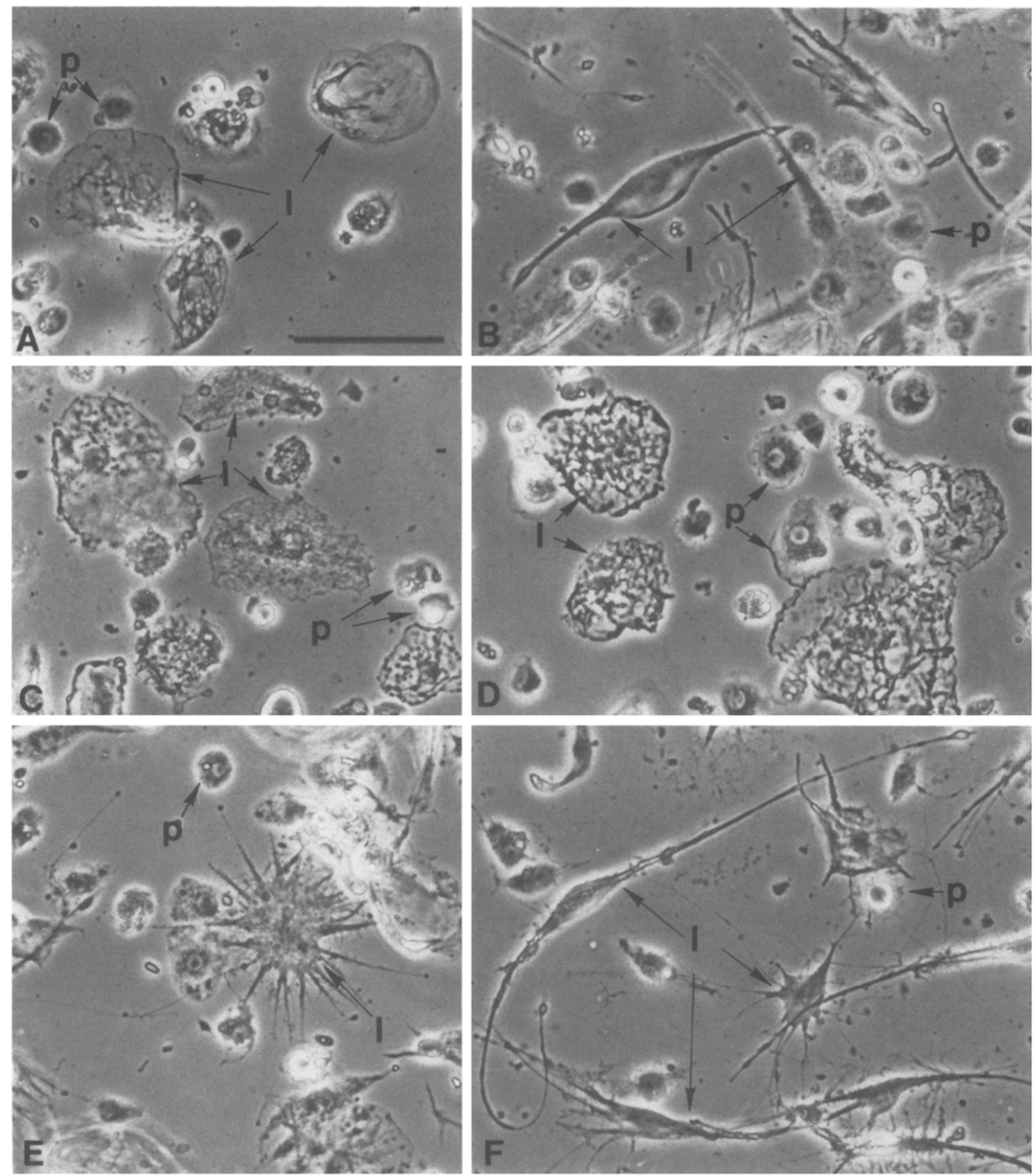

Figure 1. Drosophila hemocytes photographed after $5 \mathrm{~h}$ in vitro. $A$ Hemocytes in buffered-saline control. 1, lamellocytes; $p$, plasmatocytes spread against the glass surface. Scale bar $=50 \mu \mathrm{m}$ for $A-F$. B Sample with lamellolysin showing the typical elongation effect resulting in a bipolar cell. The two ends of the lamellocyte indicated by the left arrow have knobs near their tips. Plasmatocytes remain unchanged from the control in $B$ and the remaining samples through $F$. C Sample with lamellolysin and VCR. The lamellocyte surfaces are crinkled and there is no apparent effect of lamellolysin. There is no difference between these cells and the cells in $D$ that were treated with vincristine alone. $E$ Sample with CYB showing the stellate configuration of a lamellocyte. $F$ Effect of simuitaneous treatment with $\mathrm{CYB}$ and lamellolysin. Compare the bipolar lamellocyte to the left with the lamellocyte in a similar position in frame $B$. Arborization is apparent in the lamellocyte located by the right arrow. The lamellocyte at the bottom of the frame shows a compound effect of arborization and elongation. 

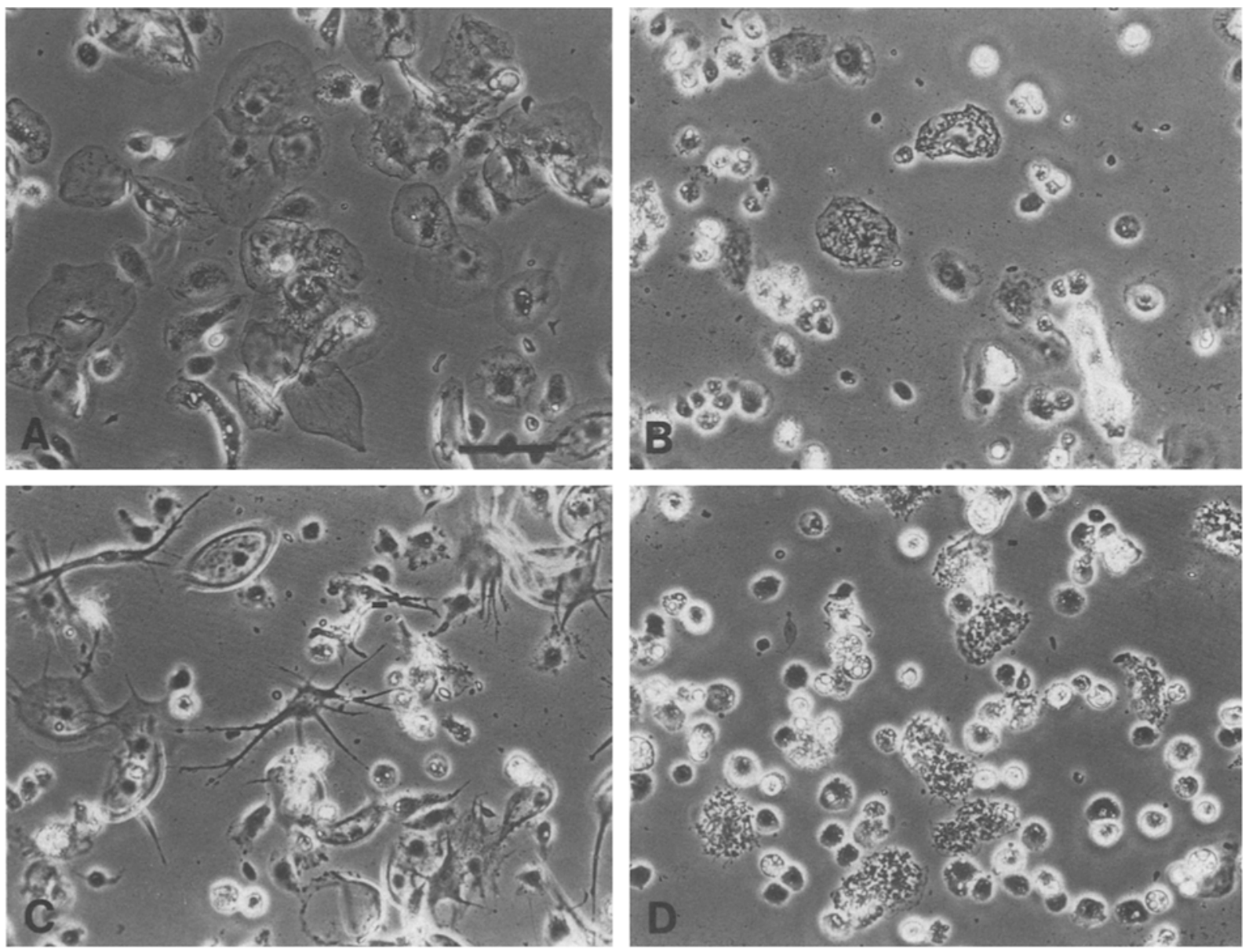

Figure 2. Hemocytes photographed after $3 \mathrm{~h}$ in vitro. $A$ Control in buffered saline. The pointed appearance of the lamellocyte at the bottom of this frame is due to folding of the cell at the two margins. $B$ Crinkled

surface of lamellocytes exposed to VBL. $C$ Arborization of lamellocytes exposed to CYB. $D$ Inhibition of the CYB effects by VBL. The crinkled surfaces of the lamellocytes are the same as in $B$. Scale bar $=50 \mu \mathrm{m}$.

some cases, the CYB-treated cells with multiple filaments and an elongated shape resembled lamellolysin-treated cells. The major elongating regions of the CYB-affected cells were often hirsute. Filamentous extensions of the CYB-affected lamellocytes had small dilations which appeared either as dark- or light-contrasting knobs under phase. Spread plasmatocytes retained their round shape in the presence of CYB.

When lamellocytes were treated with both CYB and reservoir fluid, elongation proceeded more rapidly than in the presence of reservoir fluid alone, and the arborization of some lamellocytes was more pronounced than in the presence of CYB alone (fig. $1 \mathrm{~F}$ ). The filaments extending from bipolar lamellocytes in these samples were much longer than in samples treated with reservoir fluid alone.

Since treatment of lamellocytes with CYB and reservoir fluid had a synergistic effect on the morphology of the lamellocytes and the lamellolysin effect can be blocked by VCR or VBL, the question arises whether the CYB effect can also be blocked by tubulin inhibitors. To answer this question, aliquots of a single, pooled hemocyte sample were dispensed to four Sykes chambers. To one

chamber, CYB and reservoir fluid were added, the second sample received CYB, the third had reservoir fluid alone, and no inhibitors were added to the final sample which served as the control. The results of these studies are shown in figure 2. The lamellocytes in the control sample were discoidal cells (fig. 2A); VBL caused crinkling of lamellocyte surfaces but no changes in the overall shape of the cells (fig. 2 B); CYB induced filamentous extensions on the lamellocytes as described above (fig. 2C); the lamellocytes treated with CYB and VBL were crinkled and discoidal (fig. 2D), resembling the cells treated with VBL alone. Thus, the development of filaments induced by CYB is inhibited by VBL.

\section{Discussion}

Both VBL and VCR, inhibitors of tubulin polymerization ${ }^{6,7}$, block the change in the morphology of Drosophila lamellocytes caused by wasp reservoir fluid. As demonstrated earlier ${ }^{1}$, this alteration in lamellocyte shape from a discoidal to a bipolar cell involves reorientation of microtubules. When the polymerization of tubulin is inhibited by VBL or VCR, the lamellocytes 
remain disk shaped in the presence of the wasp factor. Therefore, the elongation process induced by wasp lamellolysin requires depolymerization and repolymerization of tubulin. This requirement for tubulin polymerization does not necessarily indicate that wasp lamellolysin directly affects tubulin polymerization to yield elongated cells. The molecular target of lamellolysin may lie elsewhere than in the microtubule component of the cell in view of the effect of the actin inhibitor CYB on lamellocyte morphology.

CYB causes arborization of Drosophila lamellocytes similar to that described for tissue culture cells ${ }^{6}$. Thus, CYB and wasp lamellolysin both cause the generation of filaments from the margins of lamellocytes. This process induced by lamellolysin generally results in bipolar cells whereas the cell shape induced by CYB is usually stellate. Higher concentrations of lamellolysin in the in vitro system were conducive to multifilamentous lamellocytes ${ }^{5}$, so additional studies using various concentrations of lamellolysin and CYB are required to more adequately compare the cell shape changes induced by CYB and lamellolysin, alone and in combined treatment. The extreme response obtained when lamellocytes were treated with both CYB and lamellolysin, and the fact that both the lamellolysin effect and the CYB effect were inhibited by VBL suggests that lamellolysin may affect a process similar to that which CYB affects to result in the changed lamellocyte shape.

If microfilaments are involved in anchoring microtubules to proteins in the cell surface as proposed by Yahara and Edelman ${ }^{11}$ or if cytoskeletal components are organized in a microtrabecular lattice as proposed by Porter and Tucker ${ }^{12}$, it follows that disruption of one of the components in the network may modify the constraints imposed by this component on the network. This reasoning can explain the effects of cytoskeletal inhibitors and lamellolysin on lamellocyte morphology. Extension of filaments from the smooth surface of the lamellocyte induced by CYB or lamellolysin requires the microtubule component of the network to be intact. In the presence of a tubulin inhibitor, the filament elongation caused by either CYB or lamellolysin does not occur. CYB behaves like a capping agent to interfere with the integrity and growth of microfilaments ${ }^{6}$. When the anchoring provided to the microtubules by the microfilaments is disrupted, microtubules are free to elongate. A stellate-shaped cell would result if filament elongation proceeded from many points at the cell surface. The shape of the lamellolysin-affected cells is generally bipolar, indicating that there are restrictions in the cell surface points from which filaments develop. A bipolar shape might arise if the binding of lamellolysin to a receptor site on the cell surface results in the movement of additional receptor sites away from this first attachment point so that receptors for lamellolysin become clustered at the opposite pole. Alternative suggestions might include the stimulation of microtubule elongation deep within the cell so that elon- gation of microtubules proceeds in opposite directions to result in a bipolar cell.

Affected lamellocytes were detected within $90 \mathrm{~min}$ after female wasps oviposited in Drosophila larval hosts ${ }^{1}$. This same time period is sufficient to produce changes in lamellocyte morphology in vitro ${ }^{5}$. Considerable internal modifications may occur within the interval between exposure to the wasp factor and the appearance of cell surface extensions. Even before filaments extend from the surface of some lamellolysin-affected cells we have noticed that the cytoplasm surrounding the nucleus shows areas of dark-contrasting materials parallel to the presumptive bipolar axis under phase optics (unpublished observations). These materials may result from the initial covert changes that later become apparent as bipolar extensions of filaments.

The discoidal lamellocyte is sensitive to the factor from the parasitoid female as well as to cytoskeletal inhibitors whereas the round plasmatocyte is not markedly altered by these agents. The distinguishing feature of the lamellocyte is its flattened shape which is stable when the cells circulate in the hemocoel of the larva and in culture medium in vitro. Plasmatocytes in vitro settled on the glass surface and flattened, but these flattened plasmatocytes did not respond like lamellocytes to lamellolysin or to the cytoskeletal inhibitors. This difference in behavior must reside in the organization of the cytoskeleton in these two cell types. Fully spread lepidopteran plasmatocytes were reported to arborize in the presence of CYB in vitro ${ }^{13}$. Whether Drosophila and dipteran plasmatocytes differ in their cytoskeletal organization from lepidopteran plasmatocytes is not known. However, spread lepidopteran plasmatocytes have a typical fibroblastic morphology ${ }^{14}$ whereas Drosophila plasmatocytes spread against a glass surface have a circular shape that superficially resembles the larger lamellocyte.

Acknowledgments. This research was supported by NSF Grant DCB8517807 to RMR and NIH Grant GM-37025 to TMR.

1 Rizki, R. M., and Rizki, T. M., Proc. natl Acad. Sci. USA 81 (1984) 6154.

2 Rizki, R. M., and Rizki, T. M., Differentiation 12 (1979) 167.

3 Weber, K., Pollack, R., and Bibring, T., Proc. natl Acad. Sci. USA 72 (1975) 459.

4 Brinkley, B. R., Fuller, G. M., and Highfield, D. P., Proc. natl Acad. Sci. USA 72 (1975) 4981

5 Rizki, R. M., and Rizki, T. M., submitted for publication.

6 Bershadsky, A. D., and Vasiliev, J. M., Cytoskeleton. Plenum Press, New York 1988.

7 Dustin, P., Microtubules, 2nd edn. Springer-Verlag, Berlin 1984

8 Rizki, T. M., and Rizki, R. M., Wilhelm Roux's Arch. 189 (1980) 197.

9 Lindsley, D. L., and Grell, E. H., Genetic Variations of Drosophila melanogaster. Carn. Inst. Wash. Publ. 627 (1968).

10 Seecof, R. L., Drosophila Inform. Serv. 46 (1971) 113.

11 Yahara, I., and Edelman, G. M., Exp. Cell Res. 91 (1975) 125.

12 Porter, K. R., and Tucker, J. B., Sci. Am. 244 (1981) 57.

13 Davies, D. H., and Preston, T. M., Devl comp. Immun. 11 (1987) 353.

14 Davies, D. H., Strand, M. R., and Vinson, S. B., J. Insect Physiol. 33 (1987) 143.

0014-4754/90/030311-05\$1.50+0.20/0

(C) Birkhäuser Verlag Basel, 1990 